\title{
Marginalized Canadians may lack information about end-of-life options
}

\author{
n Cite as: CMAJ 2018 December 17;190:E1487-8. doi: 10.1503/cmaj.109-5692
}

Posted on cmajnews.com on Nov. 27, 2018.

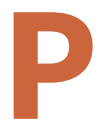

roviders of medical aid in dying (MAiD) approve most requests for the service, but marginalized Canadians may be unaware it's an option, according to information presented at the Family Medicine Forum in Toronto.

It's been two years since Canada legalized MAiD, but national data on the rollout are still lacking, according to Dr. Ellen Wiebe, a clinical professor of family medicine at the University of British Columbia. At the Family Medicine Forum, she shared insights from a survey of providers and interviews with marginalized Canadians that indicated there may be a split in access to assisted death.

Wiebe surveyed members of the Canadian Association of MAID Assessors and Providers about 742 requests they handled in 2017 that resulted in 410 deaths, or more than $20 \%$ of assisted deaths in Canada that year. The study isn't representative, she cautioned, as most of the cases were from Ontario, Manitoba and BC, but it still provides insight about overlooked aspects of MAiD. For example, "no one was looking at refusals other than Quebec," or tracking patients' reasons for requesting assisted death.

The providers in Wiebe's survey approved most patients for MAiD, refusing only 107 cases, or less than $15 \%$ of requests. In most refused cases, patients lacked the capacity to make the decision (39.3\%) or natural death was not foreseeable (36.3\%). Mental health was a factor in $10 \%$ of refusals.

More people sought assisted death because of a loss of ability (30.5\%) than because of suffering related to illness $(28.2 \%)$ or a desire for autonomy

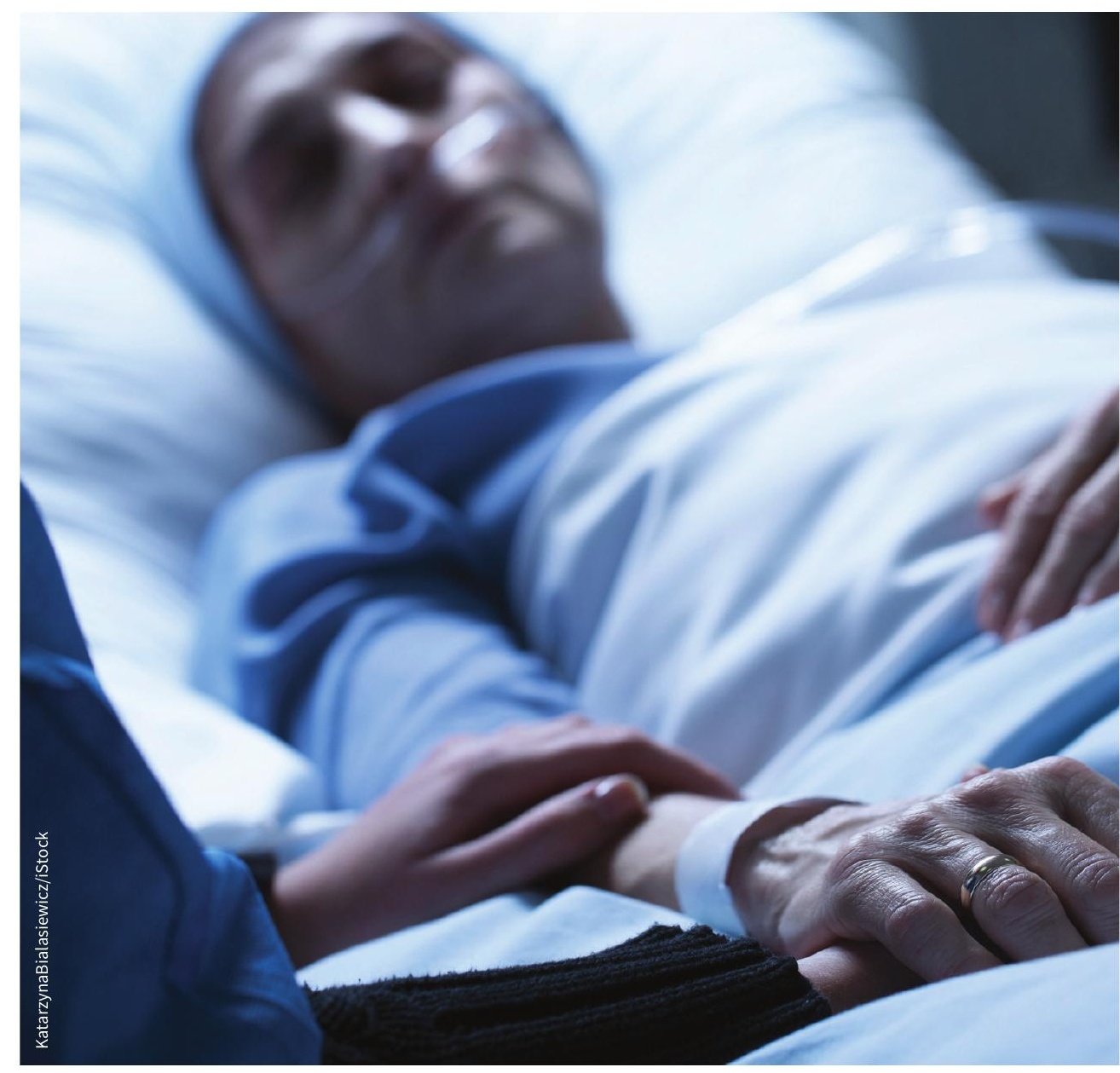

Some Canadians are unaware they have the option of medical aid in dying.

(17.8\%). Among those who received MAiD, nearly half died at home and most had health professionals and family by their side.

Providers also reported deaths taking place in hospital, hospices, clinics, hotels and at the beach, as well as in the company of friends, spiritual leaders and pets. In 51 cases, there were rituals at the time of death, including chanting, prayer, singing and toasts. Most provid- ers reported feeling positive about the deaths they assisted, although 1 in 10 reported mixed feelings.

These accounts echo Wiebe's experience as a MAiD provider. "I didn't see any big surprises." However, the picture becomes more complicated when speaking to patients from the margins of society.

Interviews with 39 marginalized people from Vancouver's downtown east side showed "very low levels of knowledge 
about palliative care, hospice and MAiD," according to Wiebe. The opioid crisis loomed large in their thoughts about death. As one person told researchers, "there was no saying goodbye" to those claimed by overdoses.

According to Wiebe, most of the people interviewed initially reported knowing nothing about MAID. With more information, "they were universal about the idea that MAiD was fine for the very, very end but not before that." They also cited stigma as a barrier to care at end of life. One person told interviewers, "They don't want to see a doctor, go to hospital, go through the humiliation of being told off because we're addicts."

Participants in the study called for more information about their options. According to Wiebe, the research team hosted an information session on the findings, distributed handouts and "teamed up with someone in the downtown eastside to provide more information."
Other MAiD providers at the Family Medicine Forum noted that they receive few requests for assisted death from marginalized populations, although it's unclear whether that's because of a difference in values or access. According to Dr. Sandy Buchman, a Toronto-based family and palliative care physician, "it's an observation that I think we are still obligated to study greatly."

Lauren Vogel, CMAJ 